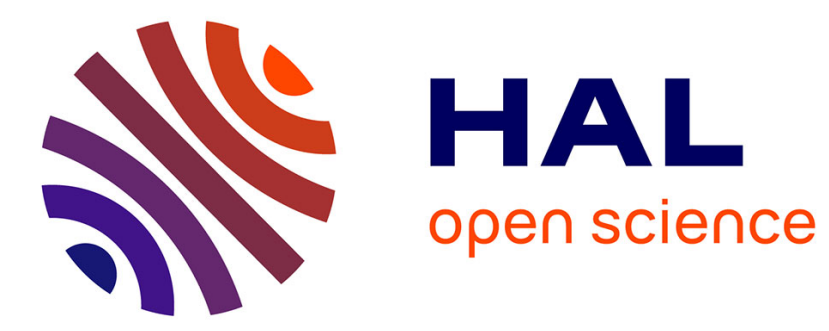

\title{
La céramique marbrée du musée de Bavai
}

\author{
Jean-Louis Boucly, Jean-Claude Carmelez
}

\section{To cite this version:}

Jean-Louis Boucly, Jean-Claude Carmelez. La céramique marbrée du musée de Bavai. Gallia - Fouilles et monuments archéologiques en France métropolitaine, 1980, 38 (2), pp.279-289. 10.3406/galia.1980.1803 . hal-01936239

\section{HAL Id: hal-01936239 \\ https://hal.science/hal-01936239}

Submitted on 14 Jun 2020

HAL is a multi-disciplinary open access archive for the deposit and dissemination of scientific research documents, whether they are published or not. The documents may come from teaching and research institutions in France or abroad, or from public or private research centers.
L'archive ouverte pluridisciplinaire HAL, est destinée au dépôt et à la diffusion de documents scientifiques de niveau recherche, publiés ou non, émanant des établissements d'enseignement et de recherche français ou étrangers, des laboratoires publics ou privés.

\section{(이) $\$$}

Distributed under a Creative Commons Attribution - NonCommercial - NoDerivatives| 4.0 


\title{
LA CÉRAMIQUE MARBRÉE DU MUSÉE DE BAVAI
}

\author{
par Jean-Louis BOUCLY et Jean-Claude CARMELEZ
}

Située au carrefour des principales routes de la Gaule du nord, la ville de Bavai fut appelée à jouer dans l'Antiquité un rôle commercial important. C'est pourquoi elle connut, dès la première moitié du $\mathrm{I}^{\mathrm{er}}$ siècle, une grande prospérité dont témoignent en particulier la quantité des céramiques importées et l'essor des officines locales de potiers. Ceux-ci, en effet, ne se contentèrent pas d'un travail routinier mais, afin de mieux résister à la concurrence et de satisfaire les goûts d'une clientèle devenue plus exigeante, ils excellèrent, en affinant leurs techniques, à imiter le bucchero, la sigillée italique, et même, lorsqu'ils furent en vogue, les vasa murrina dont parle Pline ${ }^{1}$.

De ces derniers des copies avaient été faites en Italie dès l'époque augustéenne, comme l'attestent les vases arétins trouvés à Mainz². Mais c'est surtout sous les règnes de Claude et de Néron qu'on en fabriqua en grand nombre à La Graufesenque. Dans la partie septentrionale de l'Empire, à Hofheim ${ }^{3}$ et à Bavai, et vers la même époque probablement, ce

1 Pline, Naturalis Historia, 33, 5 et 37, 18.

2 F. Oswald et T. D. Pryce, An introduction to the study of terra sigillata, ed. aug. par Grace Simpson, Londres, 1966, p. 218.

3 E. Ritterling, Das Frühromische Lager bei Hofheim, dans Ann. des Vereins für Nassauische Altertumskunde und Geschichtsforschung, Wiesbaden, 1913, p. 270-271. genre de décor fut appliqué à la vaisselle ordinaire. Cependant, si les potiers de Hofheim s'inspirèrent directement des produits du sud de la Gaule, dont ils imitèrent le vernis jaune veiné de rouge, ceux de Bavai créèrent des revêtements originaux d'une étonnante qualité.

De ces terres cuites marbrées nerviennes dont l'existence fut révélée par des fouilles fortuites en 1959, ainsi que de celles du sud de la Gaule conservées au Musée de Bavai, nous voudrions faire ici l'inventaire. D'autres monographies de ce genre préciseront ultérieurement l'importance du commerce et de l'artisanat bavaisiens dans l'Antiquité.

La sigillée marbrée du sud de la Gaule. De la sigillée marbrée fabriquée à $\mathrm{La}$ Graufesenque pendant les années 40-69 ap. J.-G. le Musée ne possède qu'un seul fragment (fig. 1, 1). C'est celui d'un bol à paroi bilobée, de la forme 27 de Dragendorff. La pâte fine, dure,

4 Le tesson, dont la provenance n'était plus connue, fut numéroté par $H$. Biévelet puis inscrit dans le registre d'inventaire ( $N$ 87). Nous en avons retrouvé la trace dans le livre d'entrée de M. Hénault, au no 2879 . Il fut découvert dans la sablière Denimal, le 21.08.1923. $\Lambda$ pparemment, le premier directeur des fouilles de Bavai n'avait jamais vu d'autres fragments de vases semblables, l'ouvrage de Hermet n'ayant paru qu'en 1934, aussi eut-il des doutes sur l'authenticité de cette pièce comme sur celle à engobe jaune qu'il ne numćrota pas. 

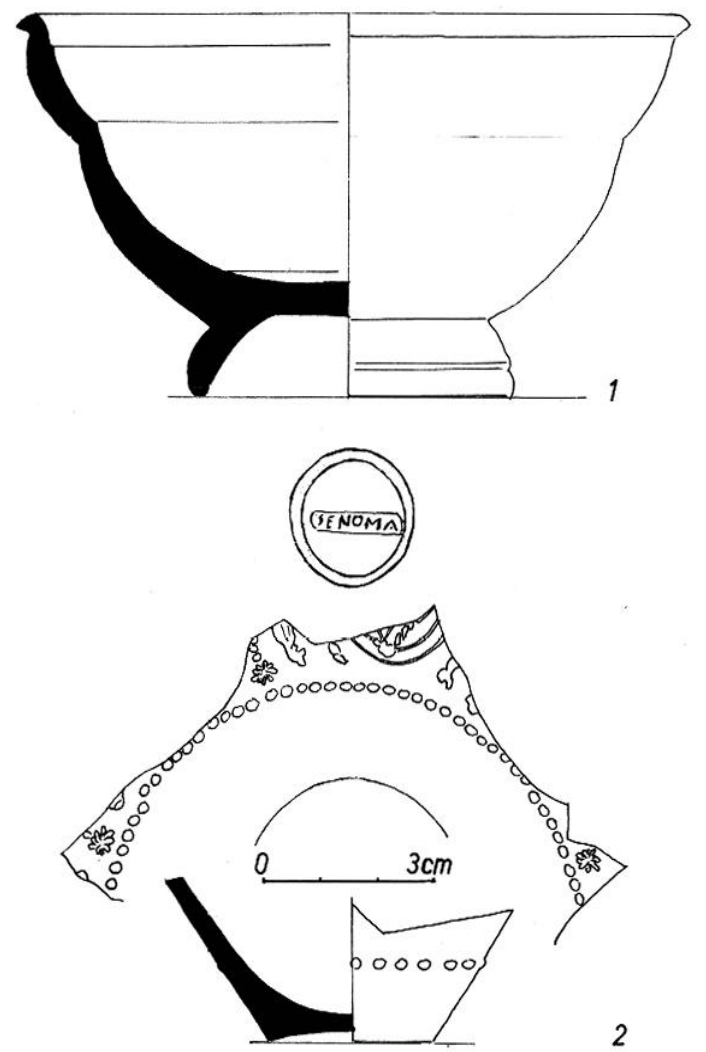

1 Céramique sigillée marbrée et à vernis jaune du sud de la Gaulc.

de couleur rouge brique, parsemée de grains de chaux, creusée de petits alvéoles, est couverte par un vernis brillant, de couleur jaune veiné de rouge brun. Près du pied, s'observent de nombreuses empreintes digitales.

Le bord, dont le diamètre extérieur est de $0,12 \mathrm{~m}$, est arrondi à sa partie supérieure, biseauté vers l'extérieur et souligné vers l'intérieur par un sillon. Sur la paroi interne $\mathrm{du}$ fond, dans un cartouche rectangulaire. étroit entouré par un sillon circulaire, est inscrite la marque SENOMA ressemblant beaucoup à celle d'un bol de Neuss ${ }^{5}$ daté des années 40-60 ap. J.-C. Or, SENO, dont l'activité à La Gaufresenque se situe pendant la période qui s'étend de Tibère à Néron, n'est cité ni par Hermet ni par Oswald ${ }^{6}$ parmi les fabricants de sigillée marbrée.

5 G. T. MARY, Die Südgallische Terra Sigillata aus Neuss, Berlin, 1967, p. 167, $\mathrm{n}^{\circ} 38$.

6 F. Hermet, La Graufesenque, Paris, Leroux,
La sigillée à vernis jaune. Au groupe des sigillées marbrées, on peut rattacher celui des vases à vernis jaune sans marbrures ${ }^{7}$. Nous n'en possédons qu'un seul fragment (fig. 1,2) dont les coordonnées archéologiques sont inconnues. Il s'agit du fond d'une petite urne sans pied de la forme 67 de Déchelette, probablement. La pâte est apparemment la même que celle du bol précédent. Le vernis luisant présente par endroits des taches plus claires et des bouillons. Le fond, un peu concave vers l'extérieur, est creusé par un sillon circulaire. La paroi évasée, d'abord lisse sur quelque $0,015 \mathrm{~m}$ de hauteur, est ensuite décorée au moule. Il ne reste malheureusement qu'une infime partie du décor, limité vers le bas par une ligne de perles rondes bien espacées. Il est possible cependant de reconnaitre les extrémités de lignes verticales séparatives, formées par de petites perles serrées et terminées par une rosette à neuf pétales ${ }^{8}$. L'espacement des lignes fait supposer qu'il y avait 5 panneaux. Sur ce qu'il reste de l'un d'eux, figurent deux tiges concentriques d'un rinceau dextrogyre. A celle du bas, s'attache un tortillon dont la ligature est épaisse et floue. A droite, et surtout à gauche du rinceau, on voit l'extrémité des pattes d'un animal dressé ou bondissant, celle d'un chien peut-être.

Ainsi donc, cet inventaire permet de compléter la carte de B. Hoffmann montrant la diffusion des céramiques marbrées rutènes ${ }^{9}$. Leur rareté dans les provinces autres que la Narbonnaise, leur absence presque complète dans la région comprise entre la Meuse, la Seine et l'Océan augmentent encore l'intérêt des découvertes bavaisiennes. En tout cas, celles-ci attestent que le commerce de la vaisselle marbrée rutène n'a pas été aussi limité qu'on pourrait le croire. Aussi peut-on penser que c'est autant pour concurrencer ces produits nouveaux que par souci de suivre la mode que certains potiers de Bavai se mirent

1934, p. 169-178. F. Oswald el T. D. Pryce, o.c., p. $219,220$.

7 F. Hermet, o.c., p. 178 : «Vases à engobe jaune *.

8 F. Hermet, o.c., no 5 , pl. 15.

9 B. Hoffmann, Les secteurs de vente de la céramique sigillee du $I^{\mathrm{er}}$ siècle de notre ère, dans Forum, $\mathrm{n}^{\mathrm{o}} 4$, 1974, p. 9-11. 
à fabriquer de la céramique gallo-belge marbrée, la plus belle qu'ils aient jamais tournée.

La céramique gallo-belge marbrée. Les céramiques gallo-belges marbrées de Bavai ont une pâte moyennement granuleuse, composée d'un mélange d'argile et de dégraissant. Celui-ci consiste en grains et lamelles de quartz assez nombreux et en fragments d'argile cuite, parfois assez gros. Une fois soigneusement tournassées, elles devaient ètre couvertes, sur la paroi interne du vaisseau pour les formes ouvertes ou sur l'autre, pour les formes fermées, par un revêtement argileux puis séchées et polies. Elles étaient cuites dans des fours à flammes nues dont certains pouvaient être pourvus de tuyaux assurant une meilleure répartition de la chaleur qui rayonnait uniformément dans la chapelle ${ }^{10}$. Aussi, en cuisson $\mathrm{A}^{11}$, leur pâte siliceuse a-t-elle pris une couleur rouge brique. Mais elle tire parfois sur le beige à cause, peut-être, d'une plus grande teneur en chaux. Quoi qu'il en soit, on reste confondu par l'habileté avec laquelle les potiers - de véritables artistes - surent tirer parti des propriétés de plusieurs revètements argileux pour imiter en perfection des variétés de marbres appréciées à leur époque. Non seulement ils sont parvenus à reproduire les couleurs nuancées de leurs modèles, mais ils ont encore rendu les veines et les marbrures particulières à ceux-ci. Nous pouvons en effet reconnaître des imitations : de marbre à fond rose parsemé de petites taches plus sombres, aux contours nébuleux, disséminées irrégulièrement ; - de marbre lie de vin, s'assombrissant par endroits en prenant des reflets violacés; - de marbre brun clair avec des taches rouge brun; - de marbre de couleur rouge sombre à reflets gris laiteux; - de marbre de couleur mastic abondamment parsemé de taches rouge brun, brillantes, aux contours irréguliers; - de marbre dont le fond de couleur mastic comporte quelques taches orange; - de marbre gris trianon avec des reflets laiteux, animé par des zébrures rouge

10 Voir, par exemple, Pro Nervia, I, 2, p. 106.

11 Terminologie de M. PIcoN, Introduction $\grave{a}$ l'élude technique des céramiques sigillées de Lezoux, Lyon, 1973, p. 65. sombre ; - de marbre gris parsemé de taches vert brun, aux contours anguleux; - de marbre où domine le rouge lie-de-vin, présentant un amalgame nébuleux de rouge sombre, de vert bronze et de gris opalescent comme l'est le test de certains coquillages.

Ces couleurs marmoréennes nuancées donnent aux céramiques gallo-belges l'apparence de sculptures un peu froides, mais précieuses et raffinées. Elles attestent en tout cas une très grande maitrise technique. Celle-ci, autant que nous pouvons en juger, fut le résultat d'une tradition artisanale locale et d'influences extérieures. Nous savons en effet que, dès l'époque augustéenne, des potiers bavaisiens cuisaient de la vaisselle luisante, grise ou noire, à reflets métallescents. Ils imitaient en outre parfaitement la couleur et le lustre des vases arétins ou des récipients en tôle de bronze. Mais si les revêtements marbrés à fond rouge, dans lesquels contrastent des teintes claires et foncées, ont pu être facilement mis au point par les potiers locaux, les autres où apparaissent plusieurs couleurs différentes nécessitèrent l'emploi de techniques originales qu'il reste à préciser. Or, la même originalité se constate dans le choix des modèles. La céramique marbrée de Bavai ne peut être en effet considérée comme une imitation des verres millefiori ni des sigillées italiques ou rutènes. Ses couleurs reproduisent fidèlement des marbres tachés et veinés dont la provenance pourrait être cherchée en Italie du nord.

Les marques. Trois fragments de fonds de plats portent une marque radiale, imprimée dans un cartouche rectangulaire. Celle du tesson numéroté $9 \mathrm{Z} 633$ est incomplète mais peut se lire [AR] ANTEDV (fig. 2, 1). Elle est en effet apposée sur des assiettes en terra rubra découvertes à Nimègue ${ }^{12}$. La reproduction d'Holwerda met en outre en évidence que ces estampilles ont été imprimées avec le même poinçon caractérisé par l'élégance des lettres, étroites par rapport à leur hauteur, et par l'écrasement de l'E entre les hastes du $\mathrm{N}$ et

12 J. H. Holwerda, De Belgische waar in Nijmegen, Nimègue, 1941, no 10, p. 140 et C.I.L., XIII, 10.010-162. 


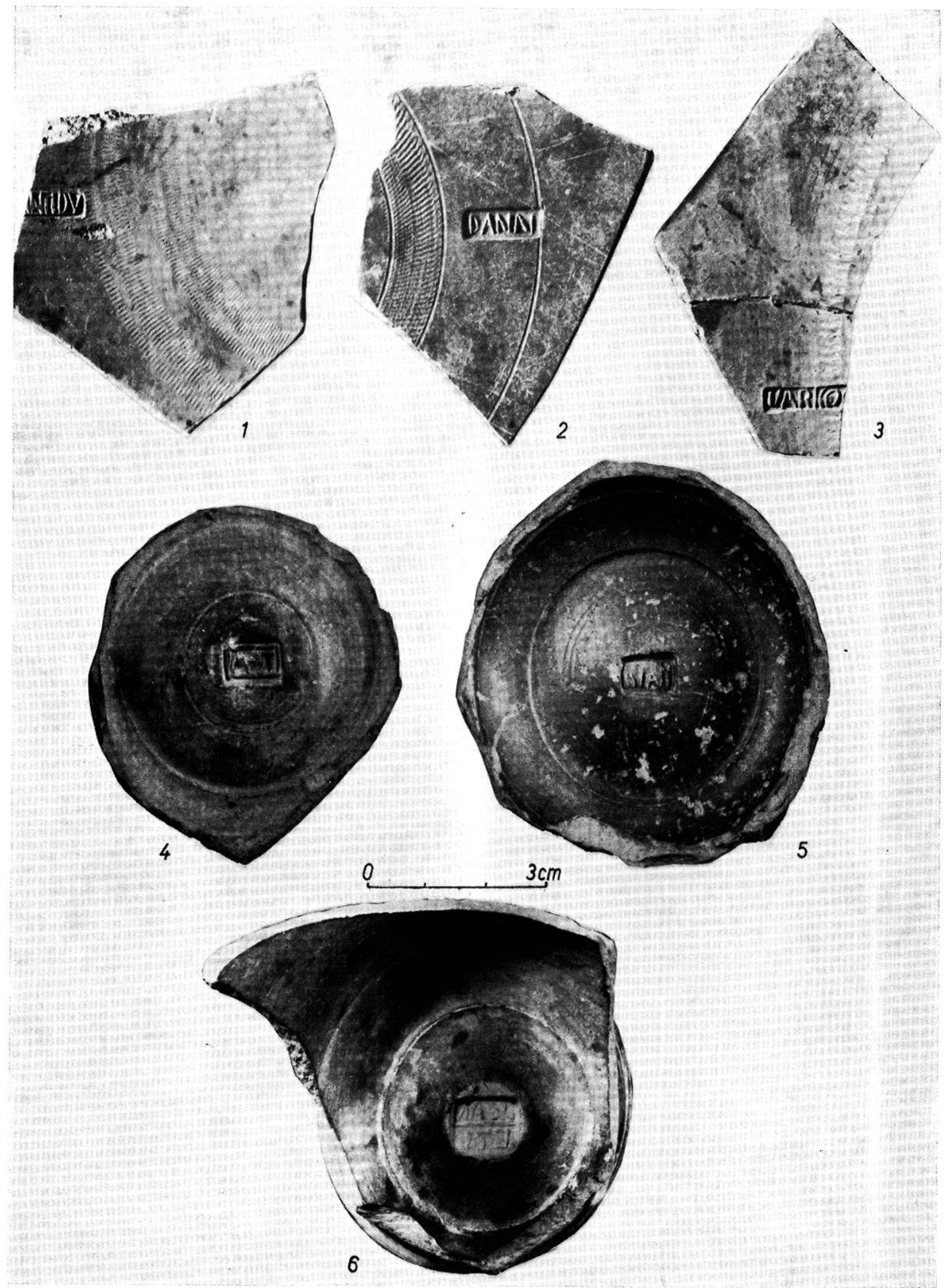

2 Marques sur céramiques marbrées de Bavai. 
du D. Douze marques d'ARANTEDV furent également découvertes dans les vestiges d'une officine de potier à Reims ${ }^{13}$. Le Corpus la signale à Épernay et au Tittelberg' ${ }^{14}$.

Le tesson inventorié $n^{0} 9 \mathrm{Z} 689$ est décoré par trois sillons circulaires concentriques. Les deux premiers limitent une bande guillochée. Entre les deux derniers est inscrite la marque DANN que Hawkes et Hull attribuent au potier DANNOMARVS connu à Poitiers, à Colchester, à Bruxelles et à Haltern ${ }^{15}$ (fig. 2, 2).

Enfin, perpendiculairement à la bande guillochée qui décore le fond de $9 \mathrm{Z} 690$, est inscrite la marque VARICOI. La dernière lettre est sinueuse; aussi ne peut-on dire s'il faut lire VARICOF ou VARICOS. Quoi qu'il en soit, la marque VARICO a été découverte à Bouy, dans la Marne, et à Colchester ${ }^{16}$. Le Corpus la signale également à Bingen $^{17}$ (fig. 2,3).

Trois autres marques sont apposées dans un cartouche rectangulaire sur le fond de tasses dont le profil ressemble à celui d'une cloche renversée, de la forme 56 de Colchester ou 103 de Hofheim ou 80 de Haltern.

Le no $9 \mathrm{Z} 455$ porte la marque AQT (A sans barre horizontale et $Q$ plus petit et placé plus haut que les autres lettres) (fig. 2,4). Cette estampille est connue à Nimègue, à Reims et à Colchester où elle est apposée sur une tasse en terra rubra de la même forme ${ }^{18}$.

Un autre tesson, assez petit et sans numéro, est inscrit BIAN, sigle inconnu, semble-t-il, ailleurs (fig. 2, 5 ).

Sur le no 9 Z 799, enfin, est apposée la marque rétrograde SCAN/ETI, inscrite sur deux lignes, d'un potier bavaisien signant de façon différente ses urnes en terra nigra (fig. 2,6). La même marque a été mal lue $\frac{\text { SCALI }}{\text { ETI }}$ àlchester où elle apparaît pendant la période VI sur

13 Gallia, XXIX, 1971, p. 295.

14 G.I.L., XIII, 10.010-744, 745.

15 C. F. C. Hawkes et M. R. HuLl, Camulodunum, First report on the excavations at Colchester, 1930-1939, Londres, p. $210, \mathrm{n}^{\circ} 72$.

16 Bouy : Gallia, XXVIII, 1969, p. 304. Colchester : o.c., no 170, pl. XLVII. La marque, qui est incomplète, n'a pas été identifiée.

17 C.I.L., XIII, 10.010-155.

18 Colchester : o.c., no 128, p. 211. - Nimègue : o.c., no 4, p. 140. - Reims : C.I.L., XIII, 10.010-155.
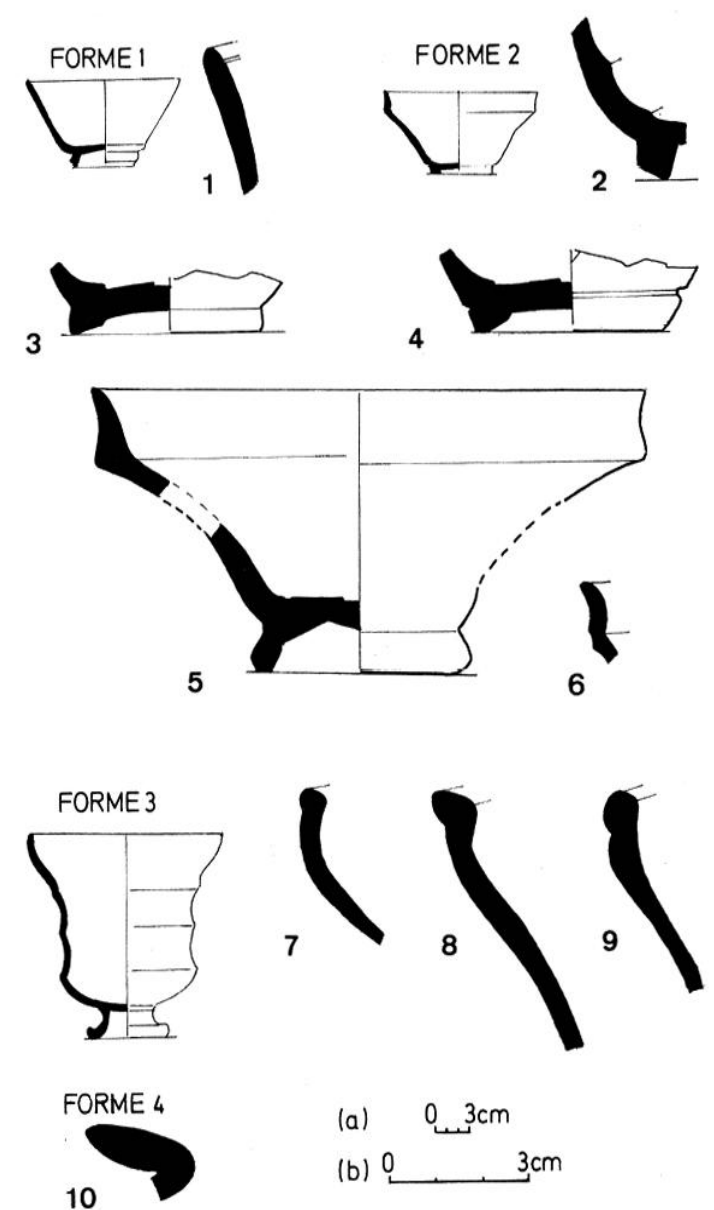

3 Bol, tasses et vases en céramiques marbrées de Bavai (a, éch. des profils de vases; b, éch. des tessons).

une tasse de la même forme ${ }^{19}$. Il faut probablement rapprocher ces timbres de celui qui figure au no 123, pl. XX de Nimègue, et que l'on peut lire SCANE. Toutes ces estampilles, dont les lettres sont belles, attestent, autant que les produits sur lesquels elles sont apposées, le souci de l'élégance et de la qualité.

Les formes. La plupart des fragments inventoriés proviennent de plats et d'assiettes mais on trouve aussi quelques débris de tasses, de bols et de vases.

Forme 1. Bol tronconique dont le bord est souligné vers l'intérieur par un sillon. Il s'agit d'une imitation de la forme 10 de Ritterling en céramique sigillée ${ }^{20}$ (fig. 3,1 ).

19 Colchester : o.c., no 138, p. 211.

20 E. Ritteriang, o.c., pl. XXXI. 
Forme 2. Tasse dont la forme rappelle celle d'une cloche renversée : Gose 301 ou Haltern 80 ou Hofheim 103 ou Colchester 56 ou Nimègue $82 \mathrm{a}^{21}$ (fig. 3, 2-6).

Forme 3. Vase caliciforme sur piédestal. La paroi moulurée s'évase à son extrémité supérieure où elle devient convexe. Le bord est biseauté vers l'intérieur. Il s'agit de la forme 75 de Colchester ou 7a de Nimègue ${ }^{22}$ (fig. 3, 7-9).

Forme 4. Vase ovoïde ou globulaire en forme de tonnelet. Le bord coudé vers l'extérieur est profilé à son extrémité (fig. 3,10 ).

Forme $5 \mathrm{~A}$. Assiette de la forme $72 \mathrm{a}$ de Haltern ou $5 \mathrm{a}$ de Colchester ou $97 \mathrm{Aa}$ de Hofheim ou 77a de Nimègue. Le bord, droit et arrondi à sa partie supérieure, surplombe la paroi interne du vaisseau et affecte vers l'extérieur la forme d'un court bandeau vertical dont la base est bien dégagée de la paroi externe. La paroi interne est moulurée en gradins et se raccorde au bord par un court marli horizontal ${ }^{23}$ (fig. 4, 11-28).

Forme 5 B. La paroi moins massive, plus oblique, a tendance à s'incurver. Il s'agit de la forme $77 \mathrm{~d}$ de Nimègue ${ }^{24}$ (fig. 4, 29-30).

Forme $5 \mathrm{C}$. Une petite moulure anguleuse raccorde le bord à la paroi interne "en gradins ». Forme 77c de Nimègue ${ }^{25}$ (fig. 4, 31-32).

Forme $6 \mathrm{~A}$. La paroi interne s'évase en formant deux parties concaves raccordées par une arête vive. Le bord, dégagé de la paroi qu'il surplombe par une gorge, est arrondi à sa partie supérieure et affecte vers l'extérieur la forme d'un court bandeau vertical. Il s'agit de la forme 72 de Haltern ou 3 de Blicquy qui est à rapprocher de Colchester $3^{26}$ (fig. 5, 33-37).

21 E. Gose, Gefässtypen der Römischen Keramik im Rheinland, Bonn, 1950. - Haltern : S. LoeschcKE, Keramische funde in Hallern, Milteilungen der AltertumsKommission für Westfalen, V, 1906, p. 101-322.

22 Nimegue : o.c., no 22 , pl. I.

23 Nimègue : o.c., no 696 , pl. XIII.

24 Nimègue : o.c., no 922 , pl. XIV.

25 Nimègue : o.c., no 921, pl. XIV.

26 Blicquy : S. J. DE LAET et H. Thoen, Etudes sur la céramique de la nécropole gallo-romaine de Blicquy (Hainaut), dans Helinium, VIII, 1968, p. 1-21.
Forme $6 \mathrm{~B}$. Le bord forme, vers l'extérieur, un bandeau convexe et saille légèrement vers l'intérieur. Forme 3 de Colchester (fig. 5, 38).

Forme $6 \mathrm{C}$. Une moulure convexe raccorde la paroi interne au fond. Ce type marque la transition entre les formes 3 et $4 \mathrm{~A}$ de Colchester (fig. 5, 39-40).

Forme 7. La paroi est à peine plus haute que le bord. Vers l'intérieur, elle s'évase légèrement et deux sillons horizontaux parallèles remplacent les ressauts que présentent les parois en gradins. Toutefois, les moulures de $9 \mathrm{Z} 631$ ont encore des arêtes vives. A rapprocher des formes 6 de Colchester, 295 de Gose, $78 \mathrm{c}, \mathrm{n}^{0} 788$, pl. XIII de Nimègue (fig. 5, 41-46).

Forme 8. Assiette à paroi très relevée et de faible hauteur. Une petite moulure raccorde le fond à la paroi interne décorée sous le bord. Celui-ci, droit et profilé à sa partie supérieure, affecte vers l'extérieur la forme d'un bandeau vertical. Haltern $72 \mathrm{Bb}$ ou Ubbergen 43,45 , pl. V ou Colchester $9^{27}$ (fig. 5, 47-51).

Forme 9. La paroi interne de l'assielte est évasée, concave et creusée par une gorge à la base du bord. Celui-ci est profilé à sa partie supérieure et affecte vers l'extérieur la forme d'un petit bandeau vertical. Nimègue $79 \mathrm{c}^{28}$ (fig. 5, 52).

Ainsi donc, les céramiques marbrées fabriquées à Bavai comprenaient une dizaine de formes au moins ${ }^{29}$ dont la plupart sont courantes durant le second quart du $\mathrm{i}^{\mathrm{er}} \mathrm{s}$. et les deux décennies suivantes. Malheureusement, il est à présent impossible de trouver, par un examen du matériel archéologique découvert cn abondance en 1959, les éléments d'une chronologie absolue.

L'entreprise Maillet, en creusant le sol pour y maçonner les fondations de bâtiments sco-

27 Ubbergen : J. BREUER, Les objels antiques découverts à Ubbergen près Nimègue, Leyde, 1931.

28 Nimegue : o.c., no 988 , pl. XIV.

29 Il s'agit de l'estimation la plus basse compte tenu de la faible surface fouillée et de la disparition, depuis 1959 , des fragments inventoriés sous les nos 241 , 288, 339, 364, 392, 405, 431, 522, 523, 527, 784 . 

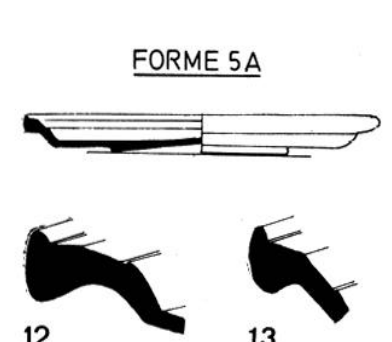

12

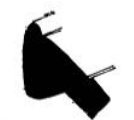

16

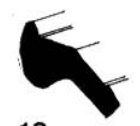

13

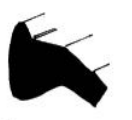

17

18
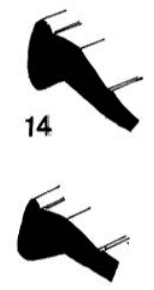

8

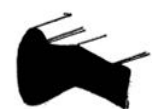

11

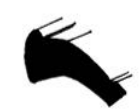

15

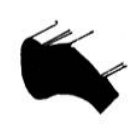

19

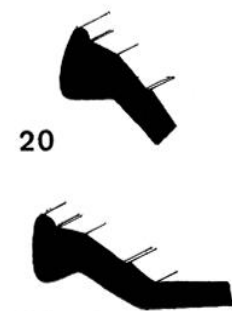

23

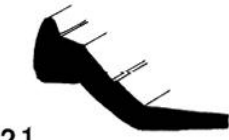

21

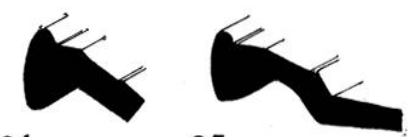

24

25

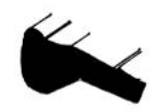

22

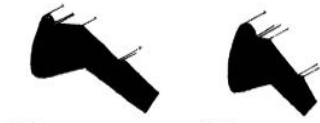

27

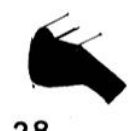

28

FORME 5B
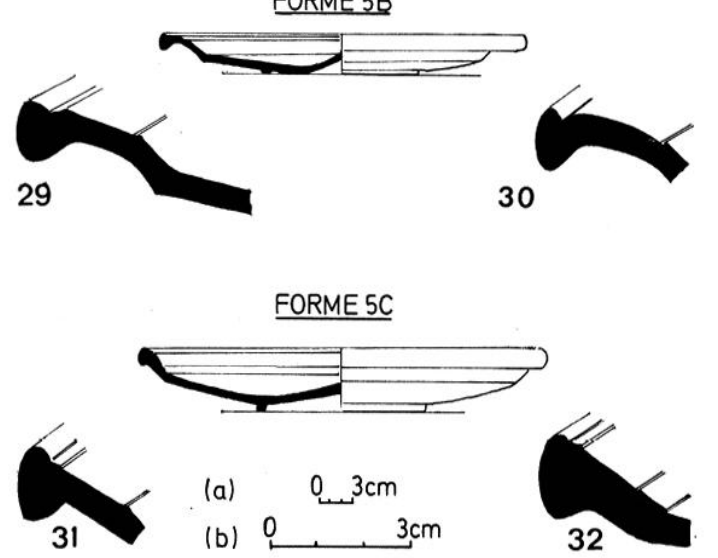

4 Assiettes et plats, formes 5 A, 5 B, 5 C (a, éch. des profils de vases; b, éch. des tessons).

laires, mit au jour fortuitement durant le mois de septembre 1959, en deux endroits plus profondément excavés n'excédant pas chacun une surface de $5 \mathrm{~m}$ sur 8 (fig. 6), un nombre exceptionnellement élevé de céramiques brisées. Elles gisaient entre moins 5 et moins $1 \mathrm{~m}$ envi-

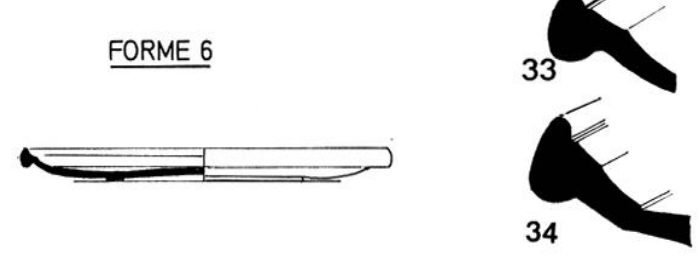

FORME 6A
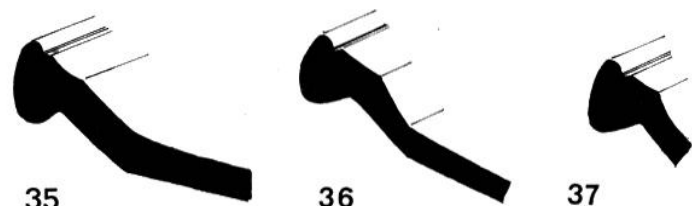

FORME6C
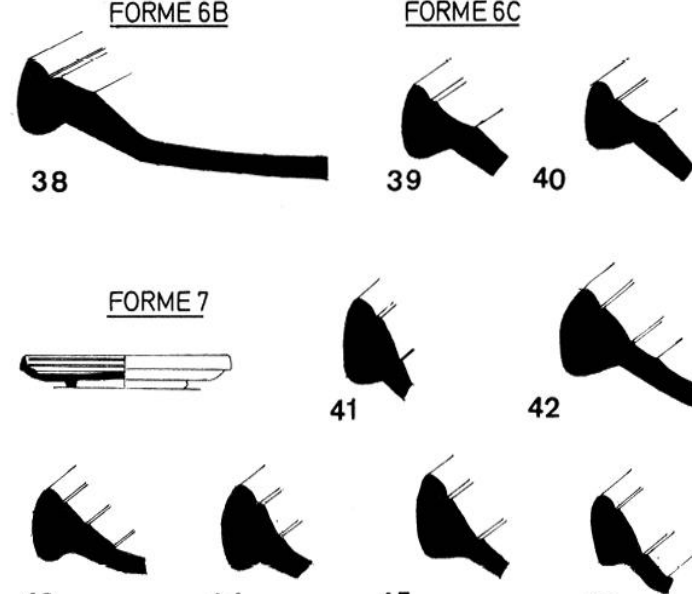

43

44
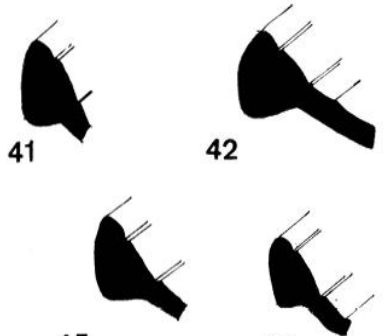

45

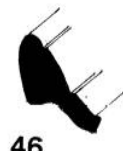

46
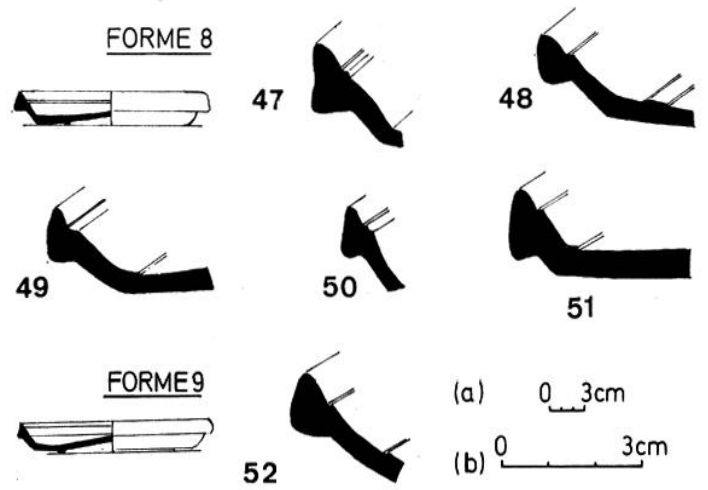

(a) $0.3 \mathrm{~cm}$

(b) $0.3 \mathrm{~cm}$

5 Assiettes et plats, formes 6 à 9 (a, éch. des proflls de vases; b, éch. des tessons).

ron dans des couches archéologiques fort nettes dans lesquelles on trouva d'autres objets tels que des tablettes à écrire, des styles, des déchets de cuir provenant d'un atelier de cordonnier, des fuseaux en bois parfaitement conservés (fig. 7). Aussitôt alerté, H. Biévelet se rendit 


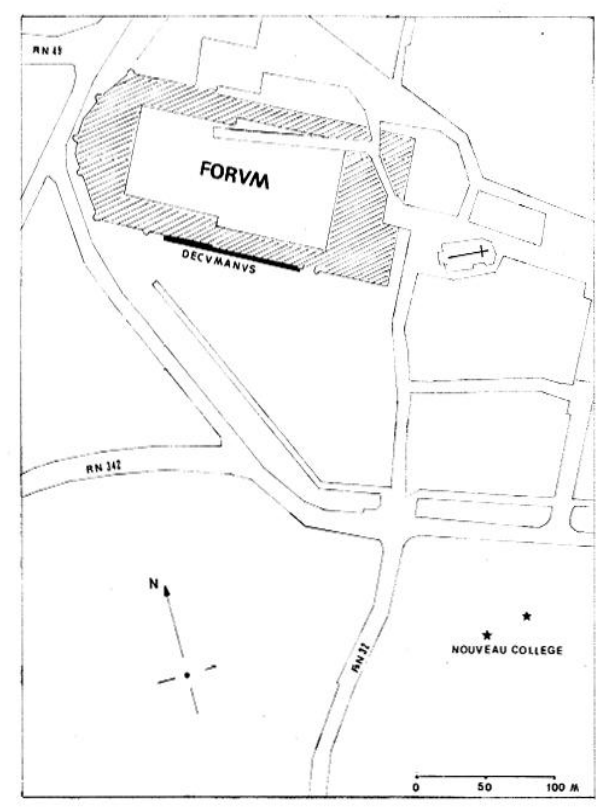

6 Plan de siluations des trouvailles de 1959.

sur le terrain transformé en bourbier par les intempéries et constata l'importance des trouvailles. En effet, à mesure que la fouille s'approfondissait, revenaient au jour de nombreuses céramiques italiques et, pour la première fois, apparaissaient des vestiges en bois, pieux et madriers de chêne, appartenant à de grandes constructions d'époque augustéenne ${ }^{30}$.

M. R. Jolin ${ }^{31}$ dessina le profil des couches archéologiques observées en coupe dans les parois de la fouille et situa sur un plan les découvertes. C'est ainsi qu'entrèrent au Musée plusieurs caisses de poteries ramassées pêlemêle. H. Biévelet numérota celles qu'il jugeait être les plus intéressantes mais conserva, contrairement à ce qu'avait fait son prédécesseur, Maurice Hénault ${ }^{32}$, les fragments non répertoriés. Leur examen nous donna la certitude que les fouilles de 1959 avaient partiellement détruit des dépotoirs d'ateliers

30 L'un de ces pieux est conservé dans le sous-sol du Musée.

31 Que H. Bievelet et R. Jolin trouvent ici l'expression renouvelée de notre cordiale gratitude.

32 Il ne s'agit nullement d'une critique. M. Hénault, Conservateur de la Bibliothèque de Valenciennes, venait une fois par semaine à Bavai. C'est alors que les ouvriers des sablières lui remettaient les tessons qu'ils avaient conservés. Généralement, seuls, les fragments inscrits ou décorés faisaient l'objet d'une transaction.

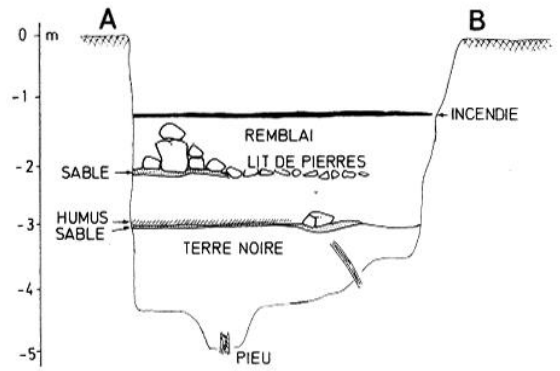

COUPES
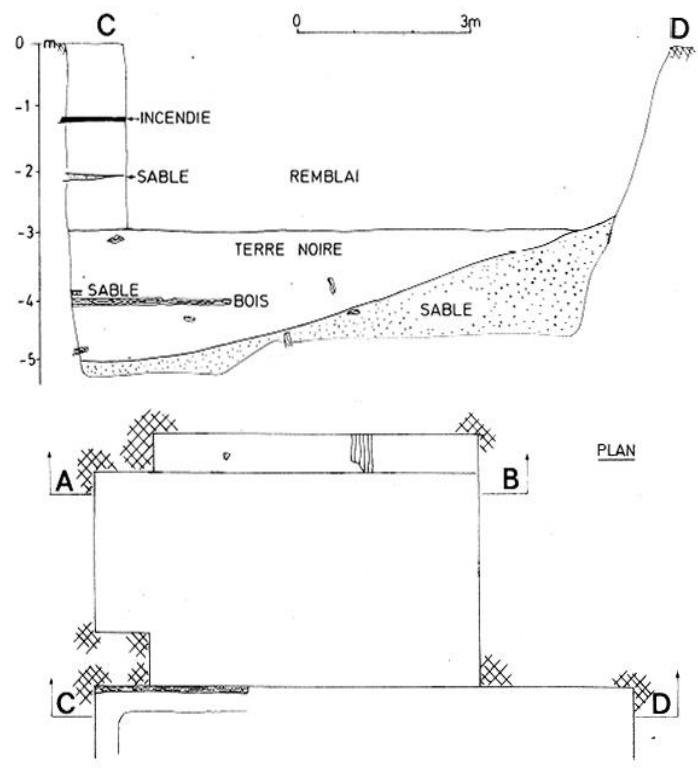

7 Relevé en coupe et en plan des vestiges observés en 1959, d'après R. Jolin.

de potiers contenant des débris de céramiques gallo-belges fabriquées dès l'époque d'Auguste. Cette supputation paraissait d'autant plus vraisemblable que les coupes de terrain dessinées en 1959 révélaient la présence de couches de sable rapporté et que le site se trouvait à la périphérie de la ville antique, à mi-pente du versant sud de la colline où abondent les sources et les gisements d'argile fictile ${ }^{33}$.

La production et la datalion des ateliers du Nouveau Collège. Si les trouvailles de 1959 ont révélé l'existence, à l'est de la chaussée de Vermand, d'officines de potiers dont nous ignorons l'importance et l'extension, les découvertes dans le forum et au sud de celui-ci de nombreuses céramiques semblables à celles

33 Surtout en direction de l'église de Louvignies où apparaissent des couches d'argile plastique grise exploitées encore au $\mathrm{XIX}^{\mathrm{e}}$ siècle. 
du Nouveau Collège attesteraient la prospérité de ces ateliers qui auraient surtout travaillé pour une clientèle locale ${ }^{34}$. Leurs produits présentent des particularités qui n'ont pas été, à notre connaissance, signalées ailleurs. Les ustensiles en terra nigra sont tantôt d'un beau noir antique luisant, irisé et métallescent par places, tantôt de la couleur grise de la plombagine, luisante parfois comme l'étain poli d'un miroir. D'autres vases, qui reproduisent la forme d'un tonnelet, se signalent par leur tournassage parfait, leur décor finement exécuté de bandes guillochées. Les plus beaux ont un revêtement brun lustré qui leur donne l'apparence de la tôle de bronze. Ainsi, les céramiques marbrées trouvent-elles leur place dans une production dont la qualité, fruit de recherches et d'innovations techniques, n'a jamais décliné pendant une période assez longue.

Sur la chronologie de ces ateliers les fouilles de 1959 et de 1972 notamment, ont apporté des renseignements non négligeables. Les premières ont livré des fragments de céramiques arétines unies. 3 d'entre elles appartiennent au service Ib, 5, au service Ic, 39 au service II, 11 au service III et 5 au service IV. En outre, les estampilles recueillies sont toutes centrales et peuvent se lire : IOT(H)VR, ATEI, $\frac{[\mathrm{G}] \mathrm{N} \text {. ATEI }}{[\mathrm{Z}] \text { ILILI }}$ Les indications chronologiques que l'on pouvait tirer d'un tel inventaire furent heureusement confirmées par la découverte en 1972 de nombreuses céramiques brisées augustéennes, tournées dans les ateliers du Nouveau Collège et associées à des céramiques lyonnaises décorées de CHRYSIPPVS ou portant le sigle $\frac{\text { MENA }}{\text { AVILI }}$ trouvé dans les dépotoirs de La Muette ${ }^{35}$. Ainsi, on peut fixer les débuts de la fabrication des terra nigra métallescentes entres les années \pm 15 et \pm 10 av. J.-C. Dans l'état présent des recherches, il semblerait que durant la première livré.

34 Aucun autre site, à notre connaissance, n'en a

35 J. LASFARGUES, Les estampilles sur sigillée lisse de l'atelier augustéen de La Muette à Lyon, Figlina, I, Lyon, 1976. Nous remercions tout particulièrement J. Lasfargues pour les renseignements qu'il nous a obligeamment communiqués. période de leur activité, les potiers du Nouveau Collège ne tournèrent que de la céramique noire ou grise. Sous les règnes de Claude et de Néron, au contraire, ils auraient diversifié leur production en fabriquant en outre de la vaisselle marbrée et des vases-tonnelets décorés. Précisons enfin que la découverte en 1959 de poteries sigillées estampillées non seulement aux noms de LABIO, de MODESTVS mais aussi de MASGVLVS, MVRANNVS, SEGVNDVS ${ }^{36}$ donnerait à penser que la production ne cessa pas complètement en 69 ap. J.-C. mais qu'elle continua, réduite à quelques types seulement d'ustensiles, au début de l'époque flavienne ${ }^{37}$.

Ainsi donc, les terres cuites marbrées paraissent avoir été fabriquées dans les ateliers du Nouveau Collège pendant les règnes de Claude et de Néron et pour une clientèle locale comme en témoignent diverses découvertes dans la sablière Mathieu ${ }^{38}$, dans le forum ${ }^{39}$ et, en assez grand nombre, au sud de ce dernier ${ }^{40}$. Quoi qu'il en soit, des 6 noms de potiers révélés par les estampilles un seul, celui de SCANETVS, est bien connu à Bavai où il est apposé sur plusieurs urnes en terra nigra. D'autres, qui signaient leurs produits VARICOF AQT, ARANTEDV eurent sûrement ou probablement des officines dans la Marne. Déplacèrent-ils ces dernières ou créérent-ils une succursale à Bavai? La question reste pour le moment sans réponse, comme le sont également celles d'une association éventuelle de ces fabricants de céramiques marbrées et de leurs rapports avec les autres potiers du Nouveau Collège.

\section{Jean-Louis Boucly et Jean-Claude Carmelez.}

36 F. Oswald, Index of Polters' stamps on terra sigillata, Margidunum, 1931.

37 Supputations que viennent confirmer les fouilles de sauvetage de 1979.

38 Le 8 juillet 1928, "une lampe en terre rouge marbrée " fut découverte dans la sablière MathieuDenimal. Inventoriée par M. Hénault sous le n 5543 , elle aussi a disparu des collections.

$393 \mathrm{Z} 19$ fut trouvé le 31 juillet 1963 par H. Biévelet dans le forum.

4015 autres tessons sont revenus au jour dans la fouille profonde exécutée à l'emplacement du nouveau Musée. 


\begin{tabular}{|c|c|c|c|c|c|}
\hline Forme & No d'inventaire & Caractéristiques & Diamètre & Figure & Marque \\
\hline 1 & $9 \mathrm{Z} 316$ & fragment de bord & 0,20 & fig. 3,1 & \\
\hline 2 & $9 \mathrm{Z} 621$ & fragment du fond et de la paroi & 0,10 & fig. 3,2 & \\
\hline 2 & $9 \mathrm{Z} 455$ & fragment de fond & 0,04 & fig. 3,3 & $\mathrm{AQT}$ \\
\hline 2 & 77 A 1 & id. & 0,045 & fig. 3,4 & BIAN \\
\hline 2 & $9 \mathrm{Z} 684$ & fragment de la paroi et du bord & 0,10 & fig. 3,5 & \\
\hline 2 & $9 \mathrm{Z} \mathrm{798,799,805}$ & fragments du bord et du fond & $?$ & fig. 1,6 & SCAN/ETI \\
\hline 3 & E 10316 & fragment de bord & 0,20 & fig. 3,7 & \\
\hline 3 & E 3958 & id. & 0,20 & fig. 3,8 & \\
\hline 3 & E 10361 & $i d$. & 0,18 & fig. 3,9 & \\
\hline 4 & $9 \mathrm{Z} 488$ & id. & $?$ & fig. 3,10 & \\
\hline $5 \mathrm{~A}$ & $9 \mathrm{Z} 569$ & $i d$. & 0,30 & fig. 4,11 & \\
\hline $5 \mathrm{~A}$ & $9 \mathrm{Z} 446$ & $i d$ & 0,30 & fig. 4,12 & \\
\hline $5 \Lambda$ & $9 \mathrm{Z} 338$ & $i d$. & 0,30 & fig. 4,13 & \\
\hline $5 \mathrm{~A}$ & $9 \mathrm{Z} 786$ & $i d$. & 0,30 & fig. 4,14 & \\
\hline $5 \mathrm{~A}$ & $9 \mathrm{Z} 192$ & id. & 0,30 & fig. 4,15 & \\
\hline $5 \mathrm{~A}$ & $9 \mathrm{Z} 202$ & $i d$. & 0,30 & fig. 4,16 & \\
\hline $5 \mathrm{~A}$ & $9 \mathrm{Z} 536$ & $i d$. & 0,30 & fig. 4,17 & \\
\hline $5 \mathrm{~A}$ & $9 \mathrm{Z} 693$ & $i d$. & 0,30 & fig. 4,18 & \\
\hline $5 \mathrm{~A}$ & $9 \mathrm{Z} 279$ & $i d$. & 0,30 & fig. 4,19 & \\
\hline $5 \mathrm{~A}$ & 9 Z 589 & id. & 0,30 & fig. 4,20 & \\
\hline $5 \mathrm{~A}$ & $9 \mathrm{Z} 228$ & $i d$ & 0,30 & fig. 4,21 & \\
\hline $5 \mathrm{~A}$ & $9 \mathrm{Z} 226$ & $i d$. & 0,30 & fig. 4,22 & \\
\hline $5 \mathrm{~A}$ & $9 \mathrm{Z} 702$ & $i d$. & 0,42 & fig. 4,23 & \\
\hline $5 \mathrm{~A}$ & $9 \mathrm{Z} 387$ & $i d$. & 0,42 & กg. 4,24 & \\
\hline $5 \mathrm{~A}$ & $9 \mathrm{Z} 539$ & $i d$. & 0,42 & fig. 4,25 & \\
\hline $5 \mathrm{~A}$ & E 5335 & $i d$. & 0,42 & fig. 4,26 & \\
\hline $5 \mathrm{~A}$ & $9 \mathrm{Z} 376$ & $i d$. & 0,24 & fig. 4,27 & \\
\hline $5 \mathrm{~A}$ & E 1199 & $i d$. & 0,24 & fig. 4,28 & \\
\hline $5 \mathrm{~B}$ & $9 \mathrm{Z} 223$ & id. & 0,30 & fig. 5,29 & \\
\hline $5 \mathrm{~B}$ & $0 \mathrm{Z} 52$ & $i d$. & 0,30 & fig. 4,30 & \\
\hline $5 \mathrm{C}$ & $9 \mathrm{Z} 716$ & id. & 0,40 & fig. 4,31 & \\
\hline $5 \mathrm{C}$ & $9 \mathrm{Z} 685$ & $i d$. & 0,30 & fig. 4,32 & \\
\hline $6 \mathrm{~A}$ & $9 \mathrm{Z} 704$ & $i d$. & 0,40 & fig. 5,33 & \\
\hline $6 \mathrm{~A}$ & $9 \mathrm{Z} 703$ & $i d$. & 0,40 & fig. 5,34 & \\
\hline $6 \mathrm{~A}$ & $9 \mathrm{Z} 284$ & $i d$. & 0,40 & fig. 5,35 & \\
\hline $6 \mathrm{~A}$ & $9 \mathrm{Z} 785$ & id. & 0,32 & fig. 5,36 & \\
\hline $6 \mathrm{~A}$ & $9 \mathrm{Z} 525$ & id. & 0,30 & fig. 5,37 & \\
\hline $6 \mathrm{~B}$ & $9 \mathrm{Z} 687$ & id. & 0,40 & fig. 5,38 & \\
\hline $6 \mathrm{C}$ & $\begin{array}{l}9 \mathrm{Z} 688 \\
\text { E } 4001\end{array}$ & id. & 0,32 & fig. 5,39 & \\
\hline 7 & $77 \mathrm{~A} 2$ & $i d$. & 0,40 & fig. 5,41 & \\
\hline 7 & $9 \mathrm{Z} 317$ & $i d$. & 0,40 & fig. 5,42 & \\
\hline 7 & $9 \mathrm{Z} 227$ & id. & 0,40 & fig. 5,43 & \\
\hline 7 & $9 \mathrm{Z} 435$ & $i d$. & 0,40 & fig. 5,44 & \\
\hline 7 & $9 \mathrm{Z} 526$ & id. & 0,32 & fig. 5,45 & \\
\hline 7 & $9 \mathrm{Z} 631$ & id. & 0,32 & fig. 5,46 & \\
\hline 8 & $9 \mathrm{Z} 447$ & $i d$. & 0,20 & fig. 5,47 & \\
\hline 8 & $9 \mathrm{Z} 283$ & & & & \\
\hline & $9 \mathrm{Z} 391$ & id. & 0,20 & fig. 5,48 & \\
\hline & $9 \mathrm{Z} 534$ & & & & \\
\hline 8 & $\begin{array}{l}9 \mathrm{Z} 201 \\
9 \mathrm{ZZ} 225\end{array}$ & $i d$. & 0,18 & fig. $5,49,50$ & \\
\hline 8 & $9 \mathrm{Z} 222$ & id. & 0,18 & fig. 5,51 & \\
\hline 9 & $9 \mathrm{Z} 664$ & id. & 0,20 & fig. 5,52 & \\
\hline$?$ & $9 \mathrm{Z} 570$ & fragment de fond & $?$ & & \\
\hline$?$ & $9 \mathrm{Z} 690$ & id. & $?$ & fig. 2,3 & VARICOF/ \\
\hline$?$ & $9 \mathrm{Z} 663$ & id. & $?$ & fig. 2,1 & /ANTEDV \\
\hline
\end{tabular}




\begin{tabular}{|c|c|c|c|c|c|}
\hline Forme & No d'inventaire & Caractéristiques & Diamètre & Figure & Marque \\
\hline$?$ & 9 Z 689 & $i d$. & $?$ & fig. 2,2 & DANN \\
\hline$?$ & E 6 & id. & $?$ & & \\
\hline$?$ & E 1163 & id. & $?$ & & \\
\hline$?$ & $3 \mathrm{Z} 19$ & id. & $?$ & & \\
\hline$?$ & $9 \mathrm{Z} 224$ & $i d$. & $?$ & & \\
\hline$?$ & $9 \mathrm{Z} 533$ & id. & $?$ & & \\
\hline$?$ & $9 \mathrm{Z} 276$ & & & & \\
\hline & $9 \mathrm{Z} 590$ & id. & $?$ & & \\
\hline & $9 \mathrm{Z} 686$ & & & & \\
\hline$?$ & $9 \mathrm{Z} 705$ & id. & $?$ & & \\
\hline$?$ & $9 \mathrm{Z} 560$ & id. & $?$ & & \\
\hline$?$ & $9 \mathrm{Z} 469$ & id. & $?$ & & \\
\hline$?$ & 9 Z 699 & id. & ? & & \\
\hline$?$ & $9 \mathrm{Z} 285$ & $i d$. & $?$ & & \\
\hline$?$ & $9 \mathrm{Z} 393$ & $i d$. & $?$ & & \\
\hline$?$ & $9 \mathrm{Z} 532$ & id. & $?$ & & \\
\hline$?$ & $9 \mathrm{Z} 445$ & id. & $?$ & & \\
\hline$?$ & $9 \mathrm{Z} 565$ & id. & $?$ & & \\
\hline$?$ & $9 \mathrm{Z} 608$ & id. & $?$ & & \\
\hline$?$ & $9 \mathrm{Z} 690$ & id. & $?$ & & \\
\hline$?$ & $9 \mathrm{Z} 538$ & id. & $?$ & & \\
\hline$?$ & E 3902 & id. & $?$ & & \\
\hline$?$ & $9 \mathrm{Z} 537$ & id. & $?$ & & \\
\hline ? & $9 \mathrm{Z} 528$ & $i d$. & $?$ & & \\
\hline$?$ & $9 \mathrm{Z} 535$ & id. & $?$ & & \\
\hline ? & $9 \mathrm{Z} 540$ & id. & $?$ & & \\
\hline$?$ & $9 \mathrm{Z} 772$ & id. & $?$ & & \\
\hline$?$ & $9 \mathrm{\Sigma} 972$ & id. & $?$ & & \\
\hline
\end{tabular}

